\title{
Microbial air monitoring in the operating theatres of Salam Center for Cardiac Surgery in Khartoum (Sudan)
}

\author{
Margherita Scapaticci \\ Ospedale di Cardiochirurgia "Salam Center" Emergency di Khartoum-Sudan
}

Key words: Operating theatre, Post-operative infections, MAM (microbial air monitoring), IMA (index of microbial air contamination), Sanitization procedures

Indagine microbiologica dell'aria nelle sale operatorie dell'ospedale di cardiochirurgia Salam Center di Khartoum (Sudan)

\section{SUMMARY}

The seriousness of postoperative infections and the increased susceptibility of patients undergoing cardiac surgery increase the demand for the operating theatre (OT) asepsis to prevent bacterial infections. In fact, the organisms carried by the air reach the wound after having sedimented onto sterile field. The air represents a critical point for quality control of air filtration systems, for sanitization procedures and for the evolution of hygienic features of the OT environment.Aim of the study is to evaluate the prevalence of microorganisms found in the operating rooms (OR) air monitoring in the Salam Center for Cardiac Surgery of Khartoum (Sudan) between July 2008 and March 2009. The specimens were collected every month in two different times: "OR at rest" (after sanitization) and "OR operational", using sedimentation method (Fisher 1972). Results showed that each sample collected at rest had IMA (index of microbial air contamination) $<5 \mathrm{CFU} /$ plt, whereas the bacterial growth was between 25 and $50 \mathrm{CFU} /$ plt when the samples had been collected in the same places during operating activities. This indicate the effectiveness of sanitization procedures and confirm that people working in OT are an important source of bacteria causing postoperative infections. Coagulase negative Staphylococci, Gram negative bacillus and Staphylococcus aureus spp. were the predominant organisms isolated.

\section{INTRODUZIONE}

I microrganismi presenti nell'atmosfera rappresentano un rischio per il malato ricoverato in ospedale esposto alla possibilità di contrarre infezioni da patogeni e opportunisti di provenienza esogena. Particolarmente a rischio sono i pazienti che vengono sottoposti ad interventi chirurgici; di conseguenza, l'aria delle sale operatorie risulta un elemento critico da monitorare per valutare i sistemi di filtrazione dell'aria, le procedure di sanificazione e le caratteristiche igienico-ambientali.

Il monitoraggio ambientale microbiologico (MAM) è destinato agli ambienti esposti a rischio biologico, ma mentre le leggi Europee ne indicano le procedure per molte matrici ambientali, non esistono, ad oggi, regole stabilite per il monitoraggio dell'aria, all'infuori della norma internazionale ISO 14698 (10). Esistono diversi metodi di campionamento (16), ma il solo ritenuto efficace per quantificare i microbi presenti nell'aria è "la conta delle unità formanti colonie", una misura dei microrganismi vivi e capaci di moltiplicarsi. I campioni di aria possono essere raccolti mediante due metodi distinti, entrambi ampiamente usati: campionamento attivo (volumetrico) e campionamento passivo (sedimentazione e impatto diretto) (10). I campionatori attivi aspirano un volume noto di aria che viene poi soffiata o deposta per impatto all'interno di una superficie agarizzata, sulla quale aderiranno i microrganismi presenti che daranno origine a colonie visibili ad occhio nudo. Il livello di contaminazione microbica viene espresso come numero di unità formanti colonie per metro cubo di aria $\left(\mathrm{UFC} / \mathrm{m}^{3}\right)$. Questi sistemi non risentono delle variazioni di velocità dell'aria entro il locale da analizzare e consentono un campionamento standardizzato per tempo e volumi di aria aspirati.

Nel campionamento passivo, invece, si espongono nell'ambiente in esame, per opportuni intervalli di tempo, piastre Petri contenenti con un idoneo terreno di coltura: su di esse si raccolgono per sedimentazione gli eventuali microrganismi veicolati dalle particelle sospese nell'aria. Dopo incubazione delle piastre per $24-48$ ore a $36 \pm 1{ }^{\circ} \mathrm{C}$, cresceranno colonie in numero proporzionale al livello di contaminazione microbiologica dell'aria e la loro conta rappresenta il numero di particelle

\section{Corresponding author: Margherita Scapaticci}

Ospedale "G. Bernabeo" - ASL Chieti Lanciano Vasto

Contrada Santa Liberata - 66026 Ortona (CH) - Tel.: 0859172218 - Cell.: 3403065670

E-mail: marghe208I@libero.it 
depositate per unità di superficie/tempo (ad esempio $\mathrm{UFC} / \mathrm{dm}^{2} / \mathrm{h}$ ) (9). Il campionamento mediante deposito per gravità è considerato un metodo di raccolta di tipo non-quantitativo.

Il volume di aria dal quale provengono le particelle è infatti sconosciuto e i risultati ottenuti non sono paragonabili con quelli ottenuti mediante altri metodi (16). Negli anni '70 Fisher definì i migliori parametri per standardizzare questo tipo di campionamento (5-8): usando piastre Petri di 9 $\mathrm{cm}$ di diametro contenenti agar sangue, dopo $24 \mathrm{~h}$ di incubazione a $37^{\circ} \mathrm{C}$ e normalizzazione dei risultati a UFC/ $\mathrm{dm}^{2}$, egli dimostrò, infatti, che la turbolenza dell'aria non aveva effetti sulla conta delle unità formanti colonie cresciute sulle piastre (6). Di conseguenza, lasciando la piastra Petri aperta all'aria per $1 \mathrm{~h}$ e posizionandola a circa 80 $100 \mathrm{~cm}$ dal pavimento e a $100-150 \mathrm{~cm}$ dal muro si ottiene un valore medio utile per valutare i microbi caduti dall'aria nell'ambiente (16). Relativamente alla sala operatoria, si ritiene che il risultato ottenuto con il campionamento passivo sia un indice clinicamente rilevante della contaminazione della ferita chirurgica (9) e il cosiddetto indice di contaminazione microbica dell'aria (IMA) è utilizzato per esprimere il grado di inquinamento microbiologico dell'aria come numero di unità formanti colonia su una piastra Petri di $9 \mathrm{~cm}$ di diametro, contenente agar nutriente (NA o PCA), lasciata aperta per un'ora, ad un metro da terra e da ogni ostacolo fisico rilevante (formula 1/1/1) (13, 16). L'utilizzo di piastre di sedimentazione, rispetto al campionamento volumetrico dell'aria, presenta il vantaggio di essere più semplice ed economico, inoltre permette una stima diretta del numero di microrganismi che si depositano sugli oggetti presenti nei luoghi monitorati. All'interno delle sale operatorie il campionamento dell'aria può essere effettuato in due modalità operative:

- con la sala operatoria vuota, pronta ad essere utilizzata per gli interventi (at rest);

- con la sala operatoria in attività (operational).

$\mathrm{Nel}$ primo caso si valuta principalmente il funzionamento dell'impianto di ventilazione e condizionamento a contaminazione controllata (VCCC); nel secondo si valuta l'osservanza delle procedure comportamentali insieme al corretto funzionamento dell'impianto VCCC.

Nell'esecuzione del monitoraggio con tecnica passiva è opportuno fare riferimento alle quattro classi di contaminazione microbica dell'aria definite dall'IMA (Tabella 1), in modo che, pur in assenza di normative ufficiali, l'operatore possa individuare la classe di contaminazione microbica dell'aria da adottare come limite massimo per l'ambiente da monitorare, in base al rischio di infezione che questo presenta.

Tabella I. Valori massimi accettabili di indici di contaminazione microbiologica dell'aria (IMA) in ambienti a rischio. (I 7). UFC/plt = unità formanti colonie/piastra.

\begin{tabular}{lc}
\hline TIPO DI REPARTO & Valori IMA (UFC/plt) \\
\hline ambienti ad altissimo rischio & 5 \\
\hline ambienti ad alto rischio & 25 \\
\hline ambienti a medio rischio & 50 \\
\hline ambienti a basso rischio & 75 \\
\hline
\end{tabular}

Considerando che, oltre all'efficienza del sistema di ventilazione, l'aumento della carica microbica dell'aria dipende dal comportamento degli operatori, il monitoraggio microbiologico, oltre a dare un riscontro oggettivo della qualità dell'aria, può costituire un utile strumento educativo per il team chirurgico.

I microrganismi la cui presenza può essere indice di pericolo di infezioni sono: Staphylococcus aureus, incluso $S$. aureus meticillino-resistente (MRSA) $(18,19)$, Clostridium difficile (11), bacilli Gram-negativi multi-resistenti (12), enterococchi vancomicina-resistenti $(12,20)$ e Aspergillus spp, quest'ultimo associato ad un rischio significativo di infezioni soprattutto in unità ospedaliere in cui sono ricoverati pazienti immunocompromessi (1).

Scopo del lavoro è stato quello di valutare la prevalenza dei microrganismi nelle tre sale operatorie (OT1, OT2, OT3) del centro di cardiochirurgia "Salam Center" di Khartoum (Sudan) nel periodo luglio 2008-marzo 2009 in condizioni operational e at rest, al fine di verificare le differenze di contaminazione nei due diversi momenti e valutare l'efficienza dei sistemi di sanificazione.

\section{MATERIALI E METODI}

I campionamenti sono stati effettuati una volta al mese in due diversi momenti: sala operatoria a riposo dopo sanificazione (at-rest), e sala operativa in attività (operational), utilizzando il metodo passivo "a sedimentazione" proposto da Fisher (58) secondo la formula $1 / 1 / 1$. I campionamenti sono stati effettuati dagli operatori, dopo aver indossato un adeguato abbigliamento da sala operatoria, essersi lavati le mani e aver indossato guanti, mediante deposizione in piastre Petri contenenti Nutrient Agar, del diametro di $9 \mathrm{~cm}$.

Le piastre sono state lasciate aperte per un'ora, ad $1 \mathrm{~m}$ da terra e ad almeno $1 \mathrm{~m}$ di distanza dalle superfici degli ostacoli circostanti (formula 1/1/1). Dopo un'incubazione in laboratorio a $37{ }^{\circ} \mathrm{C}$ per 24-48 h, è stata effettuata la conta delle colonie per ciascuna piastra. Le colonie sono state isolate mediante sottocolture su terreni TSA (Tryptone Soy Agar), Agar Mc Conkey e Agar Sale Mannite e, successivamente, identificate con metodiche 
biochimiche. La presenza di funghi è stata valutata mediante piastre SDA (Sabourad chloramphenicol dextrose agar), incubate per 7 giorni a $27^{\circ} \mathrm{C}$ e l'identificazione, secondo le metodiche standard, in base alle loro caratteristiche morfologiche macroscopiche e microscopiche (4). Il numero totale dei campioni è stato raggiunto con 91 prelievi at rest e 97 at operational, in cinque punti ritenuti critici all'interno di ciascuna sala operatoria: il tavolo operatorio, il carrello degli strumentisti, il tavolo servitore, il carrello per anestesia, il carrello dei perfusionisti.

\section{RISULTATI}

Lo scopo del campionamento è stato determinare il numero di UFC/piastra, corrispondente all'indi-

Tabella 2. Range di crescita ed identificazione dei microrganismi isolati dalle piastre utilizzate per i campionamenti mensili di aria nelle tre sale operatorie in condizioni at rest e operational. OTI = sala operatoria $I$, OT2 = sala operatoria 2 , OT3 = sala operatoria 3 , CoNS = stafilococchi coagulasi negativi, MRSA = stafilococco aureo meticillino resistente, MSSA = stafilococco aureo meticillino sensibile, $C P=$ carrello dei perfusionisti, $C A=$ carrello degli anestesisti, CS = carrello degli strumentisti, $T O=$ tavolo operatorio, $T S=$ tavolo servitore

\begin{tabular}{|c|c|c|c|c|c|c|c|}
\hline \multicolumn{4}{|c|}{ OTI at rest } & \multirow[b]{2}{*}{ Prelievi } & \multicolumn{3}{|c|}{ OTI operational } \\
\hline Prelievi & $\begin{array}{l}\text { Range di } \\
\text { crescita } \\
\text { (UFC) }\end{array}$ & $\begin{array}{l}\text { Microrganismi } \\
\text { di interesse } \\
\text { identificati }\end{array}$ & $\begin{array}{l}\text { Sede di } \\
\text { rilevamento } \\
\text { microrganismi } \\
\text { di interesse }\end{array}$ & & $\begin{array}{l}\text { Range di } \\
\text { crescita } \\
\text { (UFC) }\end{array}$ & $\begin{array}{l}\text { Microrganismi } \\
\text { di interesse } \\
\text { identificati }\end{array}$ & $\begin{array}{l}\text { Sede di } \\
\text { rilevamento } \\
\text { microrganismi } \\
\text { di interesse }\end{array}$ \\
\hline $\begin{array}{l}\text { Luglio- } \\
\text { Agos } 08\end{array}$ & $\mathrm{I}-3$ & Aspergillus spp & $\mathrm{CP}, \mathrm{CS}$ & $\begin{array}{l}\text { Luglio- } \\
\text { Agos } 08\end{array}$ & $13-22$ & CoNS & TO \\
\hline Set 08 & $1-2$ & $\begin{array}{l}\text { MSSA + bacilli } \\
\text { Gram negativi }\end{array}$ & CS & Set 08 & $3-14$ & $\begin{array}{l}\text { Bacilli } \\
\text { Gram negativi }\end{array}$ & $\mathrm{CA}$ \\
\hline Ott 08 & $0-2$ & - & & Ott 08 & $2-28$ & CoNS & $\mathrm{CP}$ \\
\hline Nov 08 & $0-3$ & - & & No v08 & $5-40$ & CoNS & TS \\
\hline Dic 08 & $0-2$ & - & & Dic 08 & $3-13$ & - & \\
\hline Gen 09 & $0-1$ & - & & Gen 09 & $0-10$ & - & \\
\hline Feb 09 & $0-1$ & - & & Feb 09 & $3-11$ & - & \\
\hline Marz 09 & $1-3$ & - & & Marz 09 & $2-6$ & - & \\
\hline & & OT2 at rest & & & & OT2 operational & \\
\hline Prelievi & $\begin{array}{l}\text { Range di } \\
\text { crescita } \\
\text { (UFC) }\end{array}$ & $\begin{array}{l}\text { Microrganismi } \\
\text { di interesse } \\
\text { identificati }\end{array}$ & $\begin{array}{l}\text { Sede di } \\
\text { rilevamento } \\
\text { microrganismi } \\
\text { di interesse }\end{array}$ & Prelievi & $\begin{array}{l}\text { Range di } \\
\text { crescita } \\
\text { (UFC) }\end{array}$ & $\begin{array}{l}\text { Microrganismi } \\
\text { di interesse } \\
\text { identificati }\end{array}$ & $\begin{array}{l}\text { Sede di } \\
\text { rilevamento } \\
\text { microrganismi } \\
\text { di interesse }\end{array}$ \\
\hline $\begin{array}{l}\text { Luglio- } \\
\text { Agos } 08\end{array}$ & $0-3$ & - & & $\begin{array}{l}\text { Luglio- } \\
\text { Agos } 08\end{array}$ & $|8-2|$ & MRSA & $\mathrm{CA}$ \\
\hline Set 08 & $0-2$ & - & & Set 08 & $6-17$ & - & \\
\hline Ott 08 & $0-3$ & - & & Ott 08 & $3-24$ & MSSA & $\mathrm{CP}$ \\
\hline Nov 08 & 0 & - & & Nov 08 & $5-52$ & $\begin{array}{l}\text { CoNS, } \\
\text { Aspergillus spp }\end{array}$ & CP, CS \\
\hline Dic 08 & $0-3$ & - & & Dic 08 & $2-6$ & - & \\
\hline Gen 09 & 0 & - & & Gen 09 & $0-3$ & - & \\
\hline Feb 09 & 0 & - & & Feb 09 & $2-13$ & - & \\
\hline Marz 09 & $0-4$ & MSSA & TS & Marz 09 & $0-6$ & - & \\
\hline & & OT3 at rest & & & & OT3 operational & \\
\hline Prelievi & $\begin{array}{l}\text { Range di } \\
\text { crescita } \\
\text { (UFC) }\end{array}$ & $\begin{array}{l}\text { Microrganismi } \\
\text { di interesse } \\
\text { identificati }\end{array}$ & $\begin{array}{l}\text { Sede di } \\
\text { rilevamento } \\
\text { microrganismi } \\
\text { di interesse } \\
\end{array}$ & Prelievi & $\begin{array}{l}\text { Range di } \\
\text { crescita } \\
\text { (UFC) }\end{array}$ & $\begin{array}{l}\text { Microrganismi } \\
\text { di interesse } \\
\text { identificati }\end{array}$ & $\begin{array}{l}\text { Sede di } \\
\text { rilevamento } \\
\text { microrganismi } \\
\text { di interesse } \\
\end{array}$ \\
\hline $\begin{array}{l}\text { Luglio- } \\
\text { Agos } 08\end{array}$ & $\mathrm{I}-3$ & Aspergillus spp & $\begin{array}{l}\text { CP, CA } \\
\text { TS, CS }\end{array}$ & $\begin{array}{l}\text { Luglio- } \\
\text { Agos } 08\end{array}$ & $\mathrm{I}-26$ & $\begin{array}{l}\text { CoNS, bacilli } \\
\text { Gram negativi }\end{array}$ & \\
\hline Set 08 & $1-2$ & - & & Set 08 & $\mathrm{I}-8$ & - & \\
\hline Ott 08 & 0 & - & & Ott 08 & $1-13$ & - & \\
\hline Nov 08 & $0-8$ & $\begin{array}{l}\text { Bacilli Gram } \\
\text { negativi }\end{array}$ & TO & Nov 08 & $3-14$ & - & \\
\hline Dic 08 & $0-3$ & - & & Dic 08 & $13-24$ & MRSA & CS \\
\hline Gen 09 & 0 & - & & Gen 09 & $5-8$ & - & \\
\hline Feb 09 & $0-1$ & - & & Feb 09 & $3-20$ & - & \\
\hline Marz 09 & $0-1$ & - & & Marz 09 & $0-4$ & - & \\
\hline
\end{tabular}


ce di contaminazione microbiologica dell'aria in quel punto (IMA) (16). I risultati ottenuti dai campionamenti sono stati interpretati facendo riferimento alle quattro classi di rischio definiti dallo standard dell'indice microbiologico dell'aria (Tabella 1), considerando le sale operatorie ambienti ad alto rischio di contaminazione e, di conseguenza, il limite IMA non superabile è stato individuato in 25 UFC per le sale operatorie in attività e in 5 UFC per le sale operatorie a riposo. I dati ottenuti (Tabella 2) hanno rivelato assenza di crescita microbica o valori di IMA $<5 \mathrm{UFC} / \mathrm{plt}$ in tutte le zone monitorate delle tre sale operatorie a riposo, con allarme in presenza di contaminazione da Aspergillus spp, mentre valori di crescita decisamente più elevati sono stati rilevati negli stessi punti durante gli interventi, con valori massimi tra i 25-52 UFC/plt: il 46\% delle piastre ha mostrato una crescita tra 0 e $5 \mathrm{UFC} / \mathrm{plt}$, il $49 \%$ tra 5 e $25 \mathrm{UFC} /$ plt, il $5 \%$ tra 25 e $50 \mathrm{UFC} /$ plt e 1 ' $1 \%$ $52 \mathrm{UFC} / \mathrm{plt}$.

In generale, le colonie batteriche cresciute nelle sale operatorie rientrano nei limiti accettabili.

Tra i microrganismi isolati dalle piastre con maggior crescita di colonie prevalgono gli Stafilococchi coagulasi negativi (59\%), seguiti da Staphylococcus aureus meticillino sensibile (23\%), MRSA (11\%), batteri Gram negativi (5\%) e Aspergillus spp (2\%).

Nelle Figure I e II vengono riportate le medie dei valori di UFC/plt ottenuti dalle piastre esposte nei punti critici durante ciascun campionamento mensile nelle tre sale operatorie in condizioni di at rest e operational.

\section{DISCUSSIONE}

La maggioranza delle infezioni chirurgiche postoperatorie in sito sono contratte al momento dell'operazione, quando c'è la possibilità che i microrganismi raggiungano la ferita aperta.

Gli interventi di cardiochirurgia sono complicati da infezioni profonde post-chirurgiche della ferita

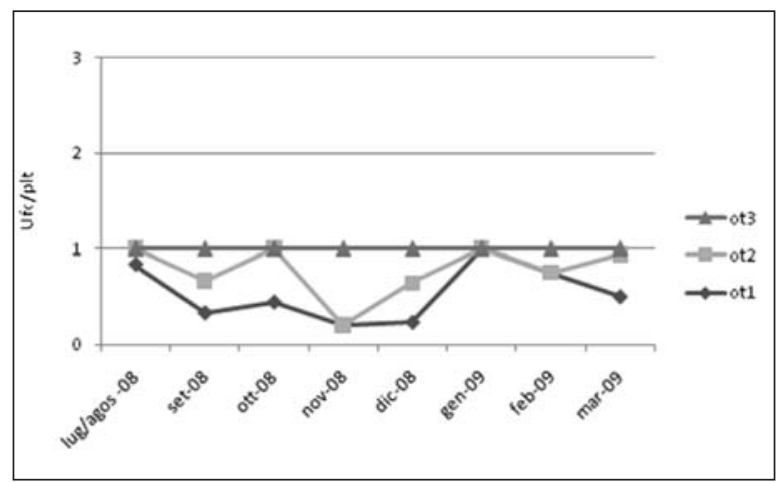

Figura I. Medie dei valori di UFC/plt ottenuti durante ciascun campionamento mensile nelle tre sale operatorie in condizioni di at rest. sternale con frequenze che vanno dallo $0.8 \%$ al $16 \%(22,23)$. Sebbene rara, questa complicazione causa considerevoli sofferenze ai pazienti ed è associata ad un elevato grado di mortalità (15).

Si ha inoltre un aumento dei costi dovuto a prolungate degenze ospedaliere, frequenti ricoveri in terapia intensiva, interventi operatori e indagini diagnostiche aggiuntivi e ulteriori trattamenti farmacologici. La maggior parte di queste infezioni è causata da cocchi Gram positivi, in particolare Staphylococcus aureus e Staphylococcus epidermidis (21). Il tentativo di ridurre l'incidenza delle infezioni postoperatorie si basa sulla conoscenza delle vie d'infezione e delle sorgenti batteriche, poiché la fonte dei microrganismi può essere esogena - da personale, oggetti inanimati, altri pazienti - oppure endogena, cioè il paziente stesso. Negli interventi chirurgici in ambiente pulito, la cute del personale di sala operatoria e dell'operando sono le fonti più importanti di microrganismi. L'aria delle sale operatorie può infatti contenere microrganismi, polvere, aerosol, lanugine, cellule epiteliali squamose della pelle e goccioline respiratorie e il livello di microrganismi presenti è direttamente proporzionale al numero di persone presenti e in movimento nella sala stessa, ai loro comportamenti nonché alle caratteristiche dell'impianto di VCCC. È quindi molto importante che in ogni struttura sia attivato un processo di formazione continua del personale per minimizzare i rischi di contaminazione ambientale e promuovere l'osservanza delle procedure comportamentali più opportune (2).

I dati ottenuti da questo studio, oltre a evidenziare l'efficacia dei processi di sanificazione - nelle sale operatorie a riposo la crescita delle colonie per piastra non supera $5 \mathrm{UFC} / \mathrm{plt}$ - confermano il fatto che la contaminazione microbica, che nelle sale operatorie si instaura con l'inizio delle attività e cresce con il passare del tempo, deriva dalle persone presenti nella sala stessa. L'aver rilevato la presenza di stafilococchi meticillino-resistenti

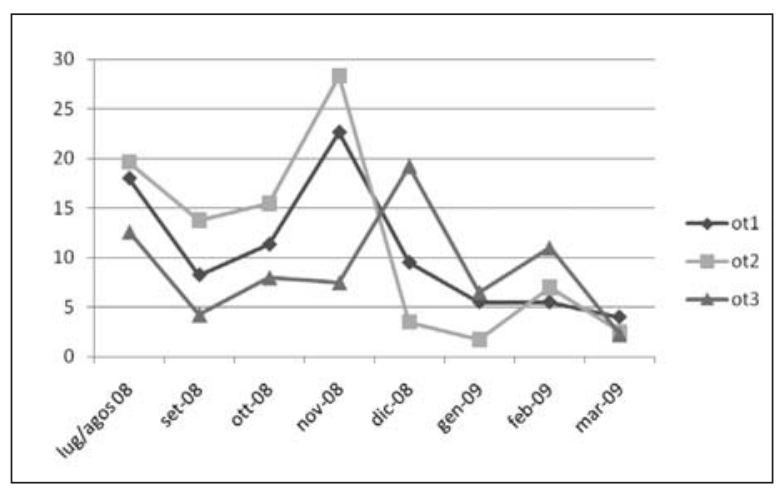

Figura II. Medie dei valori di UFC/plt ottenuti durante ciascun campionamento mensile nelle tre sale operatorie in condizioni operational. 
impone, inoltre, una più accurata pulizia dei locali, un più stretto controllo del personale in transito nel blocco operatorio e una migliore sensibilizzazione degli operatori sanitari al rischio infettivo.

\section{CONCLUSIONI}

La determinazione della carica microbica dell'aria nella sala pronta rappresenta un sistema per valutare se l'insieme delle misure di prevenzione del rischio microbiologico (sia impiantistiche, che organizzative e comportamentali) sono attive e, soprattutto, se vengono applicate correttamente.

I risultati di tale determinazione possono inoltre essere utilizzati nell'ambito di programmi di formazione del personale al fine di aumentare la consapevolezza degli operatori sugli effetti della loro non adesione agli standard comportamentali (13). Essendo stato documentato che il grado di infezioni causate da stafilococchi coagulasi-negativi è più basso tra i pazienti quando il numero di persone che si muovono nella sala operatoria durante le procedure chirurgiche è limitato (3), l'ideale sarebbe, dunque, minimizzare il traffico del personale durante l'operazione.

\section{BIBLIOGRAFIA}

1. Alberti C, Bouakline A, Ribaud P, et al. Asperigillus Study Group. Relationship between environmental fungal contamination and the incidence of invasive aspergillosis in haematology patients. $J$ Hosp Infect 2001; 48: 198-206.

2. Ayliffe GAJ. Role of the environment of the operating suite in surgical wound infection. Rev Infect Dis 1991; 13 (suppl): S800-S804.

3. Choux M, Genitori L, Lang D, Lena G. Shunt implantation: reducing the incidence of shuntinfection. $J$ Neurosurg 1992; 77: 875-80.

4. de Hoog GS, Guarro J, Gené J, Figueras MJ. Atlas of clinical fungi. 3rd ed. Utrecht: Centraalbureau voor Schimmelcultures; 2009.

5. Fisher G, Fodré S, Nehéz M. Das Ergebnis der Untersuchungen zur Feststellungs von Gesamtkeimzahl- Grenzwerten in der Luft von Operationsraumen. Z Ges Hyg 1972; 18: 729-33.

6. Fisher G, Fodré S, Nehéz M. Neuere Beitrage zur Standardisierung mit mikrobiologischen Sedimentations Luftuntersuchungen. Z Ges Hyg 1972; 18: 267-72.

7. Fisher G, Fodré S, Nehéz M. Ueber bakteriologische Untersuchungen der luft in Kindereinrichtungen unter besonderer Beruecksichtigung der GesamtkeimzahlGrenzwerte. Z Ges Hyg 1972; 18: 586-9.
8. Fisher G, Fodré S, Nehéz M. Versuche zur Feststellung von Gesamtkeimzahl-Grenzwerten in der Raumluft von Gesundheitseinrichtungen $\mathrm{Z}$ Ges Hyg 1971; 17: 576-9.

9. Friberg B, Friberg S, Burman LG. Inconsistent correlation between aerobic bacterial surface and air counts in operating rooms with ultraclean laminar air flows: proposal of a new bacteriological standard for surface contamination. J Hosp Infect 1999; 42: 287-93.

10. ISO 14698-1. Cleanrooms and associated controlled enviroments-biocontamination control. Part 1: General principles and methods. Milan: UNI; 2003.

11. Kaatz GW, Gitlin SD, Schaberg DR, et al. Acquisition of Clostridium difficile from the hospital environment. Am J Epidemiol 1988; 127: 1289-94.

12. Lemmen SW, Hafner H, Zolldann D, Amedick G, Lutticken R. Comparison of two sampling methods for the detection of Gram-positive and Gram-negative bacteria in the environment: moistened swabs versus Rodac plates. Int J Hyg Environ Health 2001; 203: 245-8.

13. Linee guida CONTARP. Il monitoraggio microbiologico negli ambienti di lavoro. Campionamento e analisi. Ed 2005.

14. Linee guida sugli standard di sicurezza e di igiene del lavoro nel reparto operatorio. ISPELS 2009.

15. Loop FD, Lytle BW, Cosgrove DM, et al. J Maxwell Chamberlain memorial paper. Sternal wound complications after isolated coronary artery bypass grafting: early and late mortality, morbidity and costo f care. Ann Thorac Surg 1990; 49: 179-87.

16. Pasquarella C, Pitzurra O, Savino A. The index of microbial air contamination. J Hosp Inf 2000; 46: 241-56.

17. Pitzurra M, Savino A, Pasquarella C. Il monitoraggio ambientale microbiologico (MAM). Ann Ig 1997; 9: 439-54.

18. Rampling A, Wiseman S, Davis L, et al. Evidence that hospital hygiene is important in the control of methicillin-resistant Staphylococcus aureus. J Hosp Infect 2001; 49: 109-16.

19. Ray AJ, Hoyen CK, Taub TF, Donskey CJ. Nosocomial transmission of vancomycin-resistant enterococci from surfaces. JAMA 2002; 287: 1400-1.

20. Rodriguez-Hernandez MJ, de Alcorn A, Cisneros JM, et al. Suppurative mediastinitis after open-heart surgery: a comparison between cases caused by Gramnegative rods and by gram-positive cocci. Clinica Microbiology and Infection 1997; 3: 523-30.

21. Ståhle E, Tammelin A, Bergström R, Hambræus A, Nyström SO, Hansson HE. Sternal wound complications-incidence, microbiology and risk factors. Eur $J$ Cardiothoracic Surg 1997; 11: 1146-53.

22. Whyte W. Sterility assurance and models for assessing bacterial contamination. J Parenter ScTechnol 1995; 40: 188-97.

23. Wong ES. The price of a surgical-site infection: more than just excess length of stay. Infect Control Hosp Epidemiol. 1999; 20: 722-4. 